\title{
Leo Testut (1849-1925)
}

\author{
Leo Testut (1849-1925)
}

Rafael Romero Reverón

ROMERO, R. R. Leo Testut (1849-1925). Int. J. Morphol., 29(4):1083-1086, 2011.

RESUMEN: Leo Testut (1849-1925), Profesor de Anatomía de la Facultad de Medicina de Lyon desde 1886 hasta 1919. Miembro de la Academia Francesa de Medicina y presidente de la Asociación Mundial de Anatomistas. Fundó y dirigió la "Revista Internacional de Anatomía y de Histología". Realizó más de 90 publicaciones sobre materias de anatomía, antropología e historia, destacándose su tratado de Anatomía Humana, publicado inicialmente en 1887 y de uso aún vigente como libro de texto y/o de consulta en muchas de las Facultades de Medicina de América Latina y de Europa.

PALABRAS ClAVE: Leo Testut; Anatomía; Anatomista; Historia; Antropología.

\section{INTRODUCCIÓN}

Leo Testut se encuentra entre los más prominentes anatomistas que hayan existido y en el área de la docencia sus obras aun constituyen una referencia importante como libros de texto y/o de consulta en el estudio de la anatomia humana en muchas Facultades de Medicina de las Universidades de América Latina y de Europa, por lo que como profesor agregado de la cátedra de Anatomía Normal, Escuela de Medicina J. M. Vargas, Facultad de Medicina, de la Universidad Central de Venezuela y como miembro correspondiente de la Sociedad Venezolana de Historia de la Medicina, me interesé en investigar y recopilar la información disponible localizada para realizar este articulo sobre algunos aspectos de interés de la vida y obra de Leo Testut, como parte de una área de investigación en desarrollo sobre personas que han realizado aportes significativos en la enseñanza y aprendizaje de la anatomia humana, como un conocimiento complementario en el estudio de la anatomía humana.

Jean Leo Testut Deynat nació en Saint Avit Senier, Francia, el 22 de Marzo en 1849, sus padres fueron Jean Testut, quien había llegado a Beaumont hacia 1840, instalando para trabajar una tienda de quincallería. Habiendo enviudado Jean Testut, se vuelve a casar con María Deynat, de cuya unión nace Jean Leo.

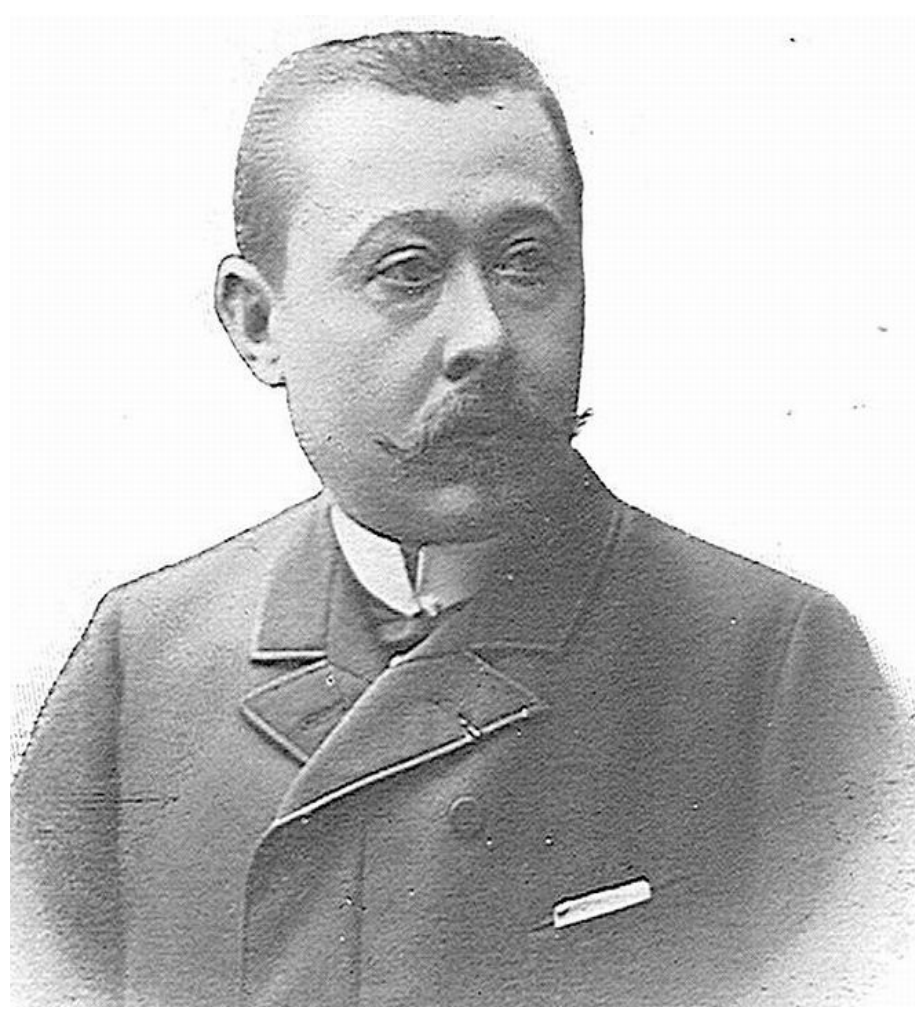

Fig. 1. Profesor Testut. Profesor de Anatomía de la Universidad de Lyon, Francia. 
Jean Leo Testut, hace sus estudios en el pequeño seminario de Bergerac y recibe la enseñanza del sacerdote Bersange, estudia el bachillerato en Sarlat. Comienza luego sus estudios de medicina en Burdeos, los cuales son interrumpidos al comenzar la guerra Franco-Prusiana de 1870 , se incorpora a las batallas y es movilizado como ayudante-mayor en el regimiento de los Móviles de Dordoña, con el doctor Barraud, en Bergeracois asisten al general de Sonis en la batalla de Loigny. Fue condecorado con la medalla conmemorativa de la Legión de honor en su carácter militar, por su coraje y patriotismo, la cual rechazó, pero que recibió posteriormente en su carácter civil la medalla de la Legión de Honor por su labor académica. Pasada la guerra, el joven Testut se reincorpora en 1878 a la escuela de Medicina de Burdeos, una vez finalizados sus estudio presenta una voluminosa tesis de doctorado titulada: "De la simetría en las afecciones de la piel: Estudio fisiológico y clínico sobre la similitud de las regiones homologas y de los órganos pares", la cual es reconocida y premiada con la medalla de Plata de la Facultad de Medicina de Paris, con la medalla de Oro de la Academia de las ciencias de Burdeos y con el premio Godard de la Academia de Medicina.

Asistió a las universidades de Burdeos y Paris entre 1872 y 1878 . En Paris complementó su formación académica en Anatomia trabajando en el laboratorio "Broca", y en los laboratorios de "Quatrefages", donde realiza estudios de antropología, así como también en el laboratorio de "Pouchet", en donde dedicó su investigación a la anatomía comparada. Leo Testut obtuvo su doctorado en la Facultad de Medicina de Paris en 1877. Fue auxiliar de Anatomía y Fisiología en la Facultad de Medicina de Burdeos de 1878 a 1884 y es nombrado Jefe de Trabajos Anatómicos en la misma Facultad (Bezerra et al., 1991).

Leo Testut contrajo matrimonio con Jeanne Clisey, quien tuvo una muerta prematura, por lo que no tuvieron descendientes. La obra de Leo Testut, es inmensa, con más de 90 publicaciones en materia de anatomía, antropología, pre-historia e historia. El doctor Leo Testut realizó muchos trabajos de anatomía comparativa, entre ellos: En 1880 escribe "Vasos y Nervios de los tejidos conjuntivos, fibrosos, serosos y óseos: anatomía y fisiología", editado en Paris, por G. Masson, el cual constituye la tesis presentada para el concurso de oposición a la cátedra de anatomía y fisiología de la Facultad de Medicina de Burdeos. En 1883 realiza el "Estudio de la longitud del flexor propio del pollicis (pulgar) en el hombre y en los monos", publicado en el Boletín de la Sociedad Zoológica de Francia. Escribe en 1884 "Las Anomalías musculares en el hombre, explicado por la anatomía com- parada, su importancia en antropología", este estudio presenta una clasificación a partir de las influencias individuales y regionales, de la herencia, de las variaciones del sistema muscular. Esta obra fue premiada por la Sociedad de Antropología de Paris con el premio Broca 1883, por el Instituto de Francia con el premio Montyon 1885 y por la Facultad de Medicina de Paris con el premio Chateauvillars 1885. En 1887 publica la separata "¿Qué es el hombre para el anatomista?", lección de apertura del curso de anatomía en la Facultad de Medicina de Lyon.

Entre 1875 y 1890 realiza entre varias investigaciones; el estudio de la apófisis epitroclear considerada como referencia quirúrgica, también realizó un estudio experimental sobre los músculos del Oso pardo de América. Realizó también artículos sobre la anatomía antropológica, entre estos: Disección de un Bosquimano, Excavaciones practicadas en las cavernas de Lavigne y Pèbousquet, Bajo Pirineos (publicado en el Boletín de la Sociedad de Borda), las anomalías musculares en los individuos de raza negra y en los individuos de raza blanca, Nuevas excavaciones realizadas en la estación de Saint-Sulpice, cantòn de Lalinde, Dordogne (publicado en la revista de la sociedad de antropología de Burdeos y el Suroeste \# 1), así como Miología y observaciones de anomalías musculares estudiadas sobre un individuo de raza negra de la isla de Borbón y también la necrópolis prehistórica de Nauthèry, en el área del cantón de Landas (publicado en el Boletín de la Sociedad de Borda).

A partir de 1883, Leo Testut en compañía del capitán Mason y de Bracquemont, excava las grutas de la estación de la Sauci, donde encuentran numerosos pedernales de cuarzo y huesos tallados del último periodo paleolítico (Ledezma, 2006).

En 1884 se establece en Lille, trabajando como profesor agregado de la Universidad de Lille, donde publica un trabajo titulado "Porción braquial del nervio musculocutáneo", el cual dio origen al epónimo "nervio de Testut".

Posteriormente desde 1886 y hasta 1919 fue profesor de la Facultad de Medicina de Lyon. El profesor Jean Leo Testut, mejor conocido por su segundo nombre Leo, se caracterizó como docente por su voz firme y pausada, por su puntualidad, y por sus precisas descripciones sobre el cuerpo humano, enriquecidas por conceptos antropológicos y filosóficos.

Publica en 1884 en el Boletín de la sociedad de antropología de Burdeos un estudio sobre la cueva del Lobo de Langlade a Saint-Amand de Belvès. A continua- 
ción estudia con Tallebois y Dufource en la región del bajo Pirineo, las sepulturas de la primera edad del Hierro.

En 1888, Leo Testut exhuma el esqueleto entero descubierto por Michael Hardy y Maurice Fèaux, el cual se encontraba sobre su lado izquierdo en posición forzada y cubierto de ocre rojo, lo que demostraba que había una sepultura intencional. Testut emprendió el estudio anatómico del esqueleto, llamado el Hombre de Chancelade, dicho estudio concluye con un informe detallado en el que indica "se trataba de un individuo de una estatura de $1,60 \mathrm{mt}$, constitución robusta y de entre 40 a 50 años de edad, el cual había vivido entre 10000 y 12000 años antes de Cristo, que tenía una gran semejanza con los esquimales del este (Encyclopædia Britannica, 2010).

Sus trabajos son publicados en el Boletín de la Sociedad Antropología de Lyon bajo el título: "Investigaciones antropológicas sobre el esqueleto cuaternario de Chancelade". Leo Testut también colabora con Alexandre Lacassagne, jefe del laboratorio de medicina forense, con el fin de solucionar tres grandes asuntos criminales de esa época, como lo fueron: el pleito de Richetto, el pleito del correo de Gouffè y el asesinato del presidente de la República de Francia, Sadi Carnot en 1894.

Publica en 1887 su tratado de anatomía humana en cuatro volúmenes, con su segunda edición en 1893, se le considera uno de los tratados de anatomía humana más completos y con una ilustración muy detallada, la mayor parte de ellas a colores dibujadas por G. Devy y S. Duprey, esta obra fue premiada por la academia francesa de medicina con el premio Saintour, 1902, es considerada una obra monumental sobre la anatomía humana, incorporado como texto para la enseñanza anatómica en la Universidad Central de Venezuela por el Dr. Pablo Acosta Ortiz en 1893 (González Guerra, 1998) y desde entonces constituye hasta la actualidad un texto básico para el estudio de la anatomía humana en esta universidad.

Este tratado de anatomía humana aun permanece vigente después de más de ciento veinte años después de su publicación inicial y continua utilizándose como libro de texto y/o de consulta en muchas de las Facultades de Medicina de América Latina y de Europa. Esta obra en su octava edición, la ultima realizada y corregida directamente por Testut constaba de 5.062 páginas, con 4.432 ilustraciones. A partir de 1910, este tratado de anatomía humana ha sido traducido del francés al italiano, al español y al alemán entre otros idiomas.

En colaboración con Octavio Jacob y H. Billet, Testut publica el Atlas de disección por regiones escrito en 1905, con 72 láminas en color y 68 figuras dibujadas por S. Duprey, es una obra muy útil como una guía que dirige e ilustra en cada momento de la disección anatómica humana.

Testut publica en 1909 su tratado de anatomía topográfica con aplicaciones médico-quirúrgicas, en dos volúmenes en colaboración con Octave Jacob con figuras dibujadas por S. Duprey, esta obra fue premiada por la Academia de Ciencias con el premio "Montyon" en 1911 y por la Academia de Medicina con el premio "Saintour" en 1912. Leo Testut fundó y dirigió el "Journal de Historia Natural De Burdeos del Sud este", los "Anales de Ciencias Naturales de Burdeos y Sud Este", así como la "Revista Internacional de Anatomía y de Histología”.

Participó como cirujano militar entre 1914 y 1915 durante la Primera Guerra Mundial, utilizando la experiencia adquirida en el campo de batalla para actualizar las ediciones posteriores de sus obras.

El doctor Leo Testut fue nombrado miembro correspondiente de la Academia Francesa de Medicina y fue también presidente de la Asociación Mundial de Anatomistas. En 1919 Testut recibe el título de profesor honorario de la Facultad de Medicina de Lyon. Se jubila en 1919, dedicándose a la labor de medico historiador, comprendiendo su obra histórica de seis volúmenes impresos y numerosos artículos publicados en revistas del sudeste de Francia, entre ellos: dos volúmenes dedicados a la historia de La Bastida de Beaumont, otros dos volúmenes dedicados a la revolución de Beaumont, un quinto volumen dedicado a la vida de la comunidad de Beaumont en el siglo XVIII y el sexto volumen dedicado a la sociedad popular de los amigos de la Constitución y su papel en Beaumont (Bezerra et al., 2008). En 1921 Leo Testut publica en conjunto con André Latarjet el Compendio de Anatomía Descriptiva.

Jean Leo Testut falleció en Caudèran, Francia el 16 de enero de 1925, a los 76 años, dejando su legado y la continuidad de su gran obra a cargo de su pupilo y sucesor, el también excelente anatomista por merito propio André Latarjet (1877-1947), quien trabajó en conjunto con Testut, posteriormente continuó la difusión de su obra, incorporando además a través de sus propia obra aportes importantes en el área del estudio de la anatomía humana.

Leo Testut es considerado uno de los más destacados anatomistas de su tiempo y su obra continúa siendo un material básico, el cual constituye un aporte significativo en el área de la enseñanza y aprendizaje de la anatomía humana. 
ROMERO, R. R. Leo Testut (1849-1925). Int. J. Morphol., 29(4):1083-1086, 2011.

SUMMARY: Leo Testut (1849-1925), Professor of Anatomy of the Faculty of Medicine of Lyon since 1886 to 1919. Member of the French Academy of Medicine and president of the International Association of Anatomists. Testut founded and directed the "International Journal of Anatomy and Histology“. He realized more than 90 publications on anatomy matters, anthropology and history, his outstanding work of Human Anatomy, first published in 1887 and even useful as textbook is used for consultation in many Medical Faculties of Latin America and Europe.

KEY WORDS: Leo Testut; Anatomy; Anatomist; History; Anthropology.

\section{REFERENCIAS BIBLIOGRÁFICAS}

Bezerra A. J. C.; Bacelar, S. S. \& Piva Júnior, L. Quem estudou no Testut? Brasília Med., 45(4):311-4, 2008.

Bezerra, A. J. C.; Didio, L. J. A. \& Piva-Junior, L. Biographical data on the anatomist Jean Leo Testut (1849-1925). Bull. Assoc. Anat., 75 (229):145-9, 1991.

Encyclopædia Britannica. Jean-Léo Testut. 2010. Disponible en: http://www.britannica.com/EBchecked/topic/588832/JeanLeo-Testut

González Guerra. M. Los estudios médicos en la Universidad Central de Venezuela a partir de 1891. Ediciones del Consejo de Desarrollo Científico y Humanístico. Caracas, Universidad Central de Venezuela, 1998. pp.30-3.

Ledezma, M. P. Huellas de un Maestro de la Anatomía Francesa Jean Leo Testut 1849-1925. Rev. Inst. Su., LXXI(128):98-105, 2006.
Dirección para correspondencia:

Dr. Rafael Romero Reverón

Servicio de Cirugía Ortopédica y Traumatología

Centro Médico Docente La Trinidad

Edif. Santa Inés, Piso 1, Control I

Caracas

VENEZUELA

Email: rafaelromeroreveron@yahoo.com.ve

Recibido : 10-05-2011

Aceptado: 04-08-2011 